\title{
SPECIFICATION OF INTEL IA-32 USING AN ARCHITECTURE DESCRIPTION LANGUAGE*
}

\author{
Jeff Bastian \\ Texas Instruments \\ 12500 TI Blvd, MS 8714 \\ Dallas, TX 75243
}

\author{
Soner Onder \\ Department of Computer Science \\ Michigan Technological University \\ Houghton, MI 49931-1295 \\ soner@mtu.edu
}

\begin{abstract}
Designing, testing, and producing a new computer processor is a complex and very expensive process. To reduce costly mistakes in hardware, the microarchitecture is usually designed and tested with the aid of a software simulator. The FAST System enables microarchitects to develop architecture simulators rapidly and is less error-prone than using a high level language such as C. In this paper, we describe how the FAST System's Architecture Description Language (ADL) has been extended to facilitate the description of complex instruction sets such as Intel's IA-32 instruction set architecture. In this respect, we demonstrate that the notion of inheritence, a key concept in object oriented programming languages can be extended for selective inheritence to enable the specification of complex instruction set architectures in architecture description languages.
\end{abstract}

Keywords: Architecture Description Language, IA32, automatic simulator generation, cycleaccurate simulators.

\section{Introduction}

Micro-architecture exploration is a difficult, error-prone and development intensive endevaour. Traditionally, there has been three distinct approaches to micro-architecture exploration; namely, hand-coding a custom simulator, generation through a hardware description language and automatic generation through an architecture description language.

Custom simulators for a specific architecture are hand-coded in a generalpurpose high-level-language, e.g. C. This group includes SimpleScalar, SuperDLX, SPIM, and URM $[2,5,3,9]$ among others. The second group in-

\footnotetext{
${ }^{*}$ This work is supported in part by a grant from DARPA, PACC Award no. F29601-00-1-0183 to the Michigan Technological University and a CAREER award (CCR-0347592) from the National Science Foundation to Soner Onder.
} 
cludes hardware description languages and simulators such as VHDL, VERILOG and ELLA [1, 12, 8, 4]. Most of these simulators are very specific to the architecture they simulate which makes it difficult to make modifications to the ISA or the microarchitecture to see how the changes affect performance. Ranging from several thousand to 30,000 lines of C code and taking 12-24 man months to develop, these are complicated software systems. Such simulators embody problems of all large scale software projects, despite the best efforts spent to increase maintainability. Trying to study such an existing simulator's source code and make changes without breaking anything can be problematic at best. Similarly, hardware description languages are not not suitable for micro-architecture exploration because they are designed to describe the hardware.

Architecture description languages on the other hand have the ability to specify the instruction set architecture (ISA), make automatic generation of support tools such as the assembler and the linker possible and hide the details of instructions from the programmer. As a result, they enable a clean model of the micro-architecture operation. More importantly, they can specify and model the operation of the micro-architecture without tying it to a particular hardware implementation and therefore seamlessly map the instruction set specification to the micro-architecture specification.

Flexible Architecture Simulation Tool (FAST) and its description language Architecture Description Language (ADL) [6] is one such system, which has been in use by a number of universities to describe and simulate micro architectures of varying complexity. Thus, FAST fills in a gap between high-level architecture-specific simulators, and low-level hardware simulators. Doing so, it allows automatic generation of the necessary system tools (assemblers, linkers, and so on) through the ADL description.

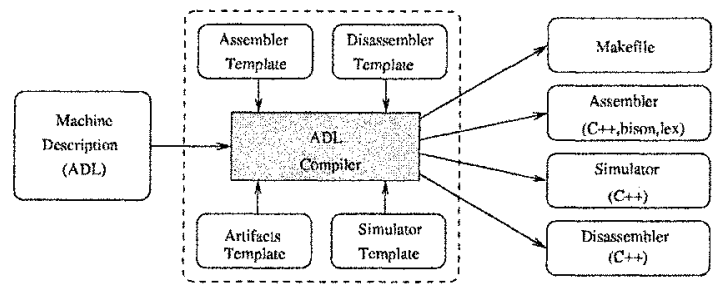

Figure 1. FAST System Components

The Flexible Architecture Simulation Tool (FAST) System shown in Figure 1 is a collection of four main components: (a) an ADL (Architecture Description Language) compiler; (b) support tools generated by the compiler (assembler, disassembler, linker, etc); (c) a cycle level simulator and debugger; (d) support tools for collecting and displaying statistics about the simulations. 
The first step is to describe the architecture in question using ADL, the Architecture Description Language. An architecture described by ADL is made up of two distinct sections - one section describes the ISA, and the other section describes how the microarchitecture works (e.g., what are the pipeline stages and what happens during each stage). The instructions are described in a declarative form, while the microarchitecture is done in an imperative form similar to other high-level imperative languages, like $\mathrm{C}$.

As an example of an instruction, consider the add instruction shown in Figure 2 from a MIPS description. The first line describes what will be seen in an assembly program - the add keyword followed by three registers. The emit line tells ADL how to translate the assembly instruction into its binary representation and consists of a series of assignments to instruction fields. This aspect of the specification is quite similar to SLED (Specification Language for Encoding and Decoding) [10,11].

The attributes section serves as the glue between the instruction set specification and the micro-architecture specification and provides the necessary abstractions for independent specification of micro-architectures from ISAs. In particular, the micro-architecture specification makes references to instruction attributes and the ISA specification maps instruction fields as well as instruction particulars to these attributes. For example, the micro-architecture specification is concerned about what is the type of the current instruction, such as arithmetic, load, store, etc., to steer the instruction to the appropriate processing unit. Similarly, the micro-architecture specification is interested in knowing the values of source and destination register numbers so that instruction execution can be modeled, but not where these fields are located in an instruction. The ISA specification conveniently maps the destination and source register attributes to the appropriate fields.

Finally, the last section describes what the instruction does during a particular pipeline phase (such as S.EX): namely, the destination register gets the results of adding the left operand to the right operand. (The operands $\mathrm{rs}$, rt, and rd are mapped to rop, lop, and dest during the decode stage of the pipeline. For an example of the microarchitecture description, let's look at a much simplified version of the instruction fetch stage of the MIPS pipeline shown in Figure 2.

During the prologue (the beginning of the clock tick), the instruction register (ir) reads an instruction from the instruction cache (icache) at the current program counter (pc), and then sets new_pc to be the next instruction, which is $p c+4$ since MIPS has fixed-length 32-bit instruction words. During the epilogue (the end of the clock tick), the current instruction and its context are delivered to the next pipeline stage and sets the new value of the program counter depending on whether or not the previous instruction was a branch instruction. ADL also includes many basic artifacts that are generic to most architectures, such as the memory and cache subsystems. Once the architecture has been 


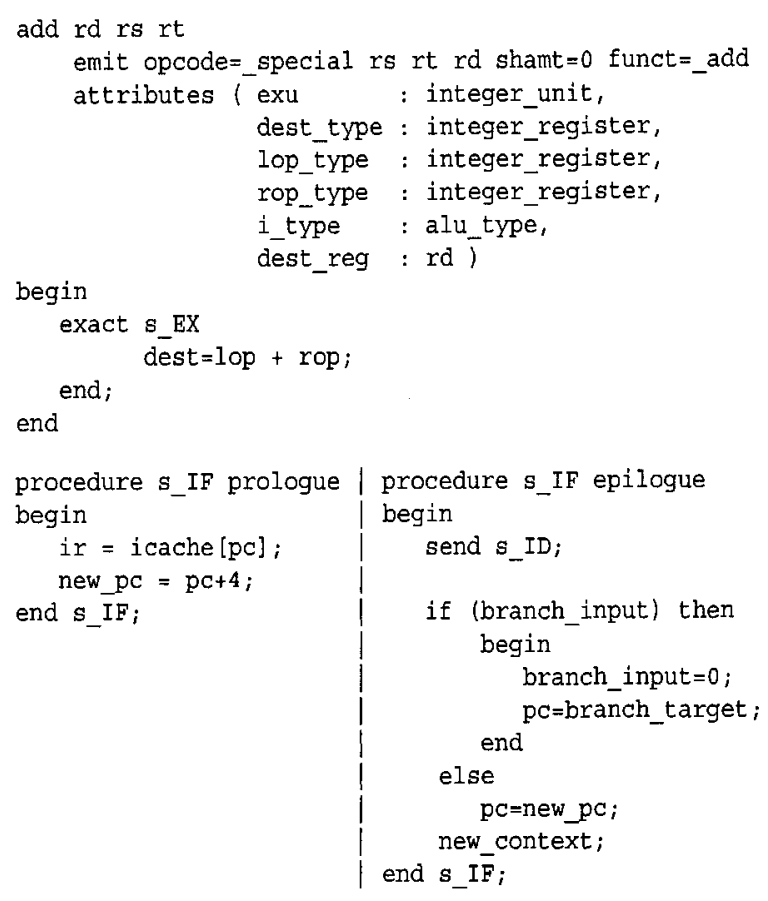

Figure 2. MIPS Instruction and Micro-architecture Specificiation in FAST/ADL

described in ADL, the file is processed by the ADL compiler. The compiler generates an assembler, a disassembler, and a simulator, as shown in Figure 1.

The FAST System was originally designed to be flexible enough to describe most modern architectures, including everything from as simple and clean-cut as a RISC processor to the most esoteric and exotic digital signal processors (DSPs). However, in early versions of the ADL compiler, certain assumptions were made and "shortcuts" taken to make it easier to implement FAST. Thus far, the MIPS architecture and a few variations on MIPS have been implemented and tested with FAST. The SPARC architecture ISA has also been specified and is in the process of being integrated with existing microarchitecture specifications. Similarly, some DSP Extensions to ADL for use with StarCore's SC-140 ISA have been described [7], but have not yet been implemented in the FAST ADL compiler. These extensions include regular expression support for addressing mode descriptions which are also useful for $\mathrm{x} 86$. 


\section{IA-32 Architecture}

The latest incarnation of IA-32 as seen in the Pentium 4 processor has its roots in the 8086 and 8088 processors from 1978. The ISA embodies a variable length instruction set encoding and the processor supports many memory models including segmented memory. The architecture also include overlapping registers. There are very few, if any, wasted bits in a typical x86 instruction. All these properties make the Intel IA-32 architecture quite challenging for an ADL specification.

In this section, we will take a closer look at these properties of IA-32 and look at how we tackled these challenges.

Variable Length Instructions: Currently FAST uses a bit numbering scheme to identify instruction fields. Although this approach is particularly appropriate for fixed length instruction formats, it is not the best approach for handling varying length instruction formats. When referencing fields in ADL, the bits are numbered right-to-left (i.e., a 32 bit word is numbered 31 to 0 , the most significant bit in position 31). With this positioning scheme, the fields can "move" in a variable length instruction set. For example, using the syntax [startbit, length], an 8-bit opcode in a 32-bit instruction is bits [31,8], but in a 16-bit instruction, the opcode moves to $[15,8]$. Furthermore, there are no special fields in ADL, including the opcode. Rather, ADL automatically distinguishes one instruction from another. The ADL compiler looks through all of the defined instructions and tries to identify a unique constant valued field for each instruction. If a series of instructions have a constant valued field but share the same value for all the instructions, they are grouped together and a second field is searched for (e.g. extended opcodes). The compiler keeps looking until it finds a unique constant valued field (or set of fields) for each instruction. This field (or set of fields) becomes the "opcode". The compiler will have the same problems as the programmer, if the fields start moving around because of their variable lengths.

An $x 86$ instruction can have up to four prefixes that modify the semantics (e.g. to use 16-bit or 32-bit registers), one or two bytes of opcode (a few reserved opcodes indicate to use the second opcode byte), a ModR/M byte for memory or register arguments, an SIB byte to help the ModR/M if necessary, and displacement and immediate fields. In other words, the fields making up the instruction format appear depending on the particular instruction format. The basic algorithm of discovering the opcode fields has to be modified so that a decoder for the architecture can automatically be synthesized.

Many Memory Addressing Modes: There are many addressing modes used in IA-32, which are, for the most part, independent of the instruction since they are encoded using the ModR/M byte (and an SIB byte if necessary). 


\begin{tabular}{|l|l|}
\hline Displacement (or Absolute) & mov \%ecx, 0xDEADBEEF \\
\hline Base & mov \%ecx, [\%esp] \\
\hline Base+Offset & mov \%ecx, [\%esp-4] \\
\hline Base+Index+Offset & mov \%ecx, [\%esp+\%edi-4] \\
\hline Base+(Index*Scale)+Offset & mov \%ecx, [\%esp+\%edi*2-4] \\
\hline Base+Index & mov \%ecx, [\%esp+\%edi*1] \\
\hline Base+(Index*Scale) & mov \%ecx, [\%esp+\%edi*2] \\
\hline Index+Offset & mov \%ecx, [\%edi*1-4] \\
\hline (Index*Scale)+Offset & mov \%ecx, [\%edi*2-4] \\
\hline
\end{tabular}

Figure 3. Intel IA-32 addressing modes

IA-32 addressing modes are shown together with examples in Figure 3. The challenging aspect of the many addressing modes in IA-32 is trying to define them succinctly in ADL, since the fields are mostly independent of the opcode. That is, the opcode alone does not indicate all of the fields that follow the opcode. For example, the mov instruction shown in the above table has the opcode of $0 \times 89$, which indicates that the opcode byte will be followed by a ModR/M byte, with the Reg/Opcode field of the ModR/M byte selecting a general purpose register. What follows the ModR/M byte, if anything, is indicated by the ModR/M byte. Thus, each byte in the instruction provides a hint as to what comes next.

The simplest way to approach this problem is to enumerate every possible variation of an instruction as if it is a separate instruction, since ADL allows instructions to be overloaded, just like functions in $\mathrm{C}++$. For example, the mov instruction overloaded for Displacement and Base addressing modes:

mov reg32 disp emit opcode $=0 \times 89$ mod $={ }^{\prime} 00^{\prime} b$ regop $=r e g 32 \mathrm{rm}=\prime^{\prime} 100^{\prime} \mathrm{b}$ disp $\ldots$

mov reg 32 base emit opcode $=0 \times 89$ mod $=100^{\prime} b$ regop=reg 32 rm=base ...

However, this leads to the problem of having to overload the same instruction many times due to the many addressing modes. There are nine addressing modes listed above, however, three modes (Base + Displacement, Base + Index + Displacement, and Base + (Index*Scale) + Displacement) can use either an 8-bit or a 32-bit displacement, giving us 12 effective modes. Furthermore, there are restrictions on when \%esp and \%ebp can be used for base or index registers. Treating these restrictions as special addressing modes (which would be necessary in the current version of ADL) gives us 6 additional special case modes, for a total of 18 addressing modes!

Creating separate ADL instruction definitions for every combination of $x 86$ opcode with addressing mode would generate thousands of ADL instructions. This is tedious and highly error-prone.

Overlapping Registers: The IA-32 architecture is $99.9 \%$ fully backwards compatible with the original $\times 86$ processor (the $0.1 \%$ difference is the addition of certain instructions that allow the processor to switch between operat- 
ing operating modes, e.g., protected mode, system management mode, or real mode). One of the features of this backwards compatibility is a set of registers that have overlapping parts.

IA-32 includes eight 32-bit general purpose registers: EAX, ECX, EDX, EBX, ESP, EBP, ESI, and EDI. However, in order to maintain backwards compatibility, there are aliases for 8-bit and 16-bit parts of the registers. For example, AL, AH, and AX all describe different parts of the EAX register. AL and $\mathrm{AH}$ are two 8-bit registers that represent the lower and upper 8 bits of the 16-bit register $\mathrm{AX}$, and $\mathrm{AX}$ is the low 16 bits of the 32-bit register EAX.

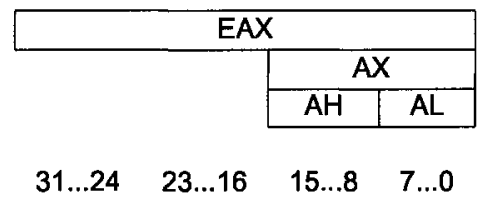

Registers ECX, EDX, and EBX are broken down in a similar fashion. Registers ESP, EBP, ESI, and EDI have aliases for the lower 16 bits SP, BP, SI, and DI respectively. As a side note, while the above eight registers are noted as general purpose, the registers occasionally have special uses, hence the seemingly obtuse names of the registers.

Mixed Arguments: Unlike RISC architectures, instructions in CISC machines can operate directly on memory. That is, RISC machines, or load-store architectures, only have two instructions that can operate on memory, load and store, while all other instructions must operate on registers. CISC instructions have no such restrictions. This leads to instructions that can have a variety of arguments. The mov instruction takes two arguments, a source and a destination, one of which must be a register, the other can be either a register or a memory address. The address can come in any one of the 18 modes listed above. In addition to creating a plethora of instructions (also described above), many instructions will need to access memory at some point during the execution which will result in a lot of replicated ADL code (i.e., every instruction description will contain the same copied-and-pasted ADL code to read from or write to memory).

Although some of these issues have been addressed within the context of instruction set specification with the SLED approach, as it can be seen, the approach taken by SLED is inadequate for automatic generation of simulators. Although one can describe x86 ISA in less than 500 lines of code in SLED, the language was only designed for encoding and decoding instructions (as the name implies). Many instructions in $\mathrm{x} 86$ are encoded/decoded the same way with the only difference being the opcode, so patterns are used to define many instructions in one line. On the other hand, in order to tie in the micro- 
architecture specification, one needs to be able to specify the semantics of each instruction. Since the semantics of each instruction are very different, attaching semantics to many opcodes cannot be done with one line, and an alternative technique must be sought.

\section{Our Solutions}

An IA-32 processor can run in many different modes. For the purposes of our work, we were able to simplify the model with a few assumptions and restrictions: (a) no segmented memory (i.e., flat address space); (b) 32-bit mode (16-bit instructions require 0x66 prefix); (c) no 36-bit Physical Address Extension (PAE) support; (d) no ISA extensions (MMX, SSE, SSE2).

We made these restrictions based on the way modern applications run on $\times 86$ platforms. Very few programs use segmented memory except the occasional device driver. 16-bit mode is only used when running old programs compiled for DOS or Windows 3.1 and earlier. 36-bit PAE mode is only used by programs with intense memory requirements on high end servers, such as large database systems that require more than 4GB of RAM. We chose to exclude MMX, SSE and SSE2 for now in order to get a framework in place; when this is done, the new instructions will be easily added with little or no changes to the framework necessary.

Variable Length Instructions: In the previous version of ADL, fields could be defined in any order since the start bit dictated where it would actually appear. This freedom is lost, but it was rarely (if ever) taken advantage of. Therefore, the problem of variable length instructions is addressed by removing the requirement that the start bit of individual fields need to be specified. Instead, the fields of an instruction now need to be specified in order and only the length of the field needs to be specified. With this scheme the compiler may look through every instruction and identify the longest instruction and use its length for a pseudo-fixed-length instruction (for $\mathrm{x} 86$, this will be 16-bytes, or 128-bits). Then the compiler can internally generate the start bits for each field based on this length (thus, for $x 86$, the opcode will always be [127,8] (note that opcode prefixes are really just special opcodes)). Undefined fields will not generate pad bits in the encoded binary; i.e., a 16-bit instruction will only have bits 127 through 111 defined, but the binary encoding of the file will not have 111 padded bits following the instruction.

Addressing Modes and Regular Expressions: The DSP Extensions use a combination of flex-style regular expressions with register variables. We will use these extensions for $\mathrm{x} 86$ as well, with the addition of typesets. The syntax is fairly straightforward as shown in Figure 4.

The regular expressions in the addressing mode define the syntax of the addressing mode, but they do not define the semantics. For example, in base_index 

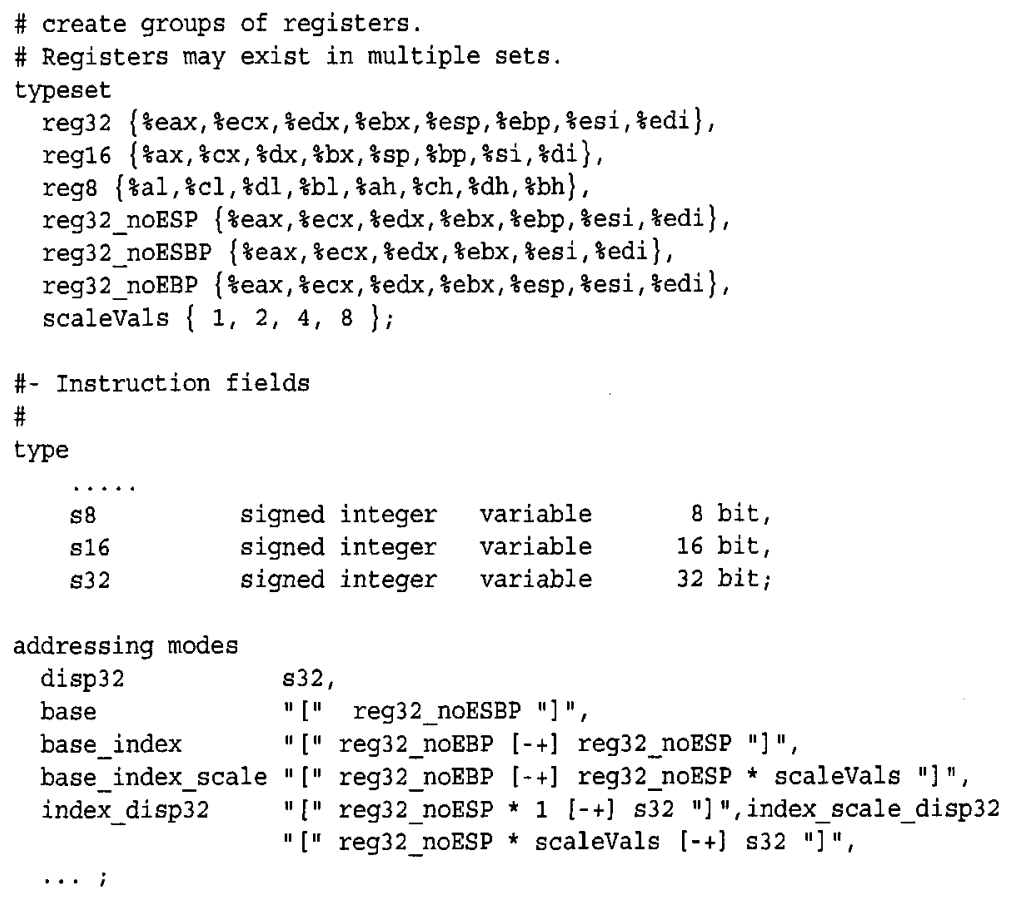

Figure 4. Use of typesets to describe addressing modes

mode above, the assembler does not know if reg32_noEBP is the base or the index; it only knows to expect a 32-bit register excluding \%ebp. Mapping the two registers to some meaningful value like base or index is handled by a user-defined procedure.

Instruction Templates: Most of the problems we encountered (overlapping registers, variable length instructions, etc.) were handled by a simple extension of ADL or modification of an existing syntax. The real problem with x86 was the combination of instructions with multiple addressing modes. With over 400 instructions and many of them using up to 18 modes for memory addressing, plus register-to-register arguments, we could easily end up with thousands of descriptions if we were to enumerate each permutation as a separate instruction.

We toyed with many ideas for reducing the number of enumerations. Although it is possible to write a wrapper program to enumerate the instructions and generate the ADL code, such a solution is specific to the instruction set at hand and defeats the purpose of using an architecture description language in the first place. What is needed is to increase the power of the ADL so that these instructions can also be specified seamlessly and naturally. Furthermore, to a 
lesser extend similar problems make RISC instruction set descriptions in ADL also somewhat longer than necessary. Therefore these language extensions can be generic enough to be useful for other architectures (e.g., making the existing MIPS implementation shorter and more concise with our new language extensions).

Our careful study of the SLED encoding scheme where patterns are extensively used lead us to believe that it may be possible to define an encoding pattern for the addressing modes and let each instruction inherit the right pattern? This turned out to be the key idea for the $\mathrm{x} 86$ extensions to ADL: treat instructions as objects and use multiple inheritance with a twist!

The encoding patterns would be defined by a series of templates, and the real instructions would inherit the properties from these patterns. The objects in ADL are the instruction templates and the instructions themselves. Templates differ from normal instructions in two ways.

Fields in instruction templates can be grouped or made optional with the use of regular-expression like syntax. Parentheses group fields together, (field1 field2 ...), to indicate that all of the fields in the group must appear together. That is, field1 cannot exist without field2, and vice-versa. This is useful for larger fields like the ModR/M byte in IA-32, which consists of three smaller fields: the 2 bit mod field, the $3 \mathrm{bit}$ reg/op field, and the $3 \mathrm{bit} \mathrm{r} / \mathrm{m}$ field, used to describe how memory and/or registers will be addressed. A '?' following a field indicates the field is optional. For example, the SIB byte is optional depending on the ModR/M byte, thus, it appears as (scale index base)? . A '?' is really just shorthand for $\{n, m\}$ syntax (where $n=0$ and $m=1$ ) which says the previous item must appear at least $n$ times but no more than $m$ times. Finally, a $\mid$ indicates logical-or, useful for fields that vary in size. Some instructions have 8-bit immediates, others 16-bit, and others 32-bit, and others none at all, so, putting it all together: (imm8 |imm16|imm32)?

Templates do not exist in the actual instruction set. That is, when the generated assembler is assembling code, it will never try to match a template with a real assembly instruction.

Templates can inherit properties from other instruction templates and override fields or sections from the parent. This allows creation of a master template. A master template is really just another template (i.e., it is not a special type of template), but it helps the programmer avoid syntactical errors. In IA32 , there is one general instruction format:

\begin{tabular}{|l|l|l|l|l|l|}
\hline Prefixes & Opcode & ModR/M & SIB & Disp & Immediate \\
\hline
\end{tabular}

In this format there may be up to 4 prefixes, where each prefix is 1 byte long. The opcode field can be 1 or 2 bytes long and is followed by the optional ModR/M and SIB bytes. Displacement and the Immediate fields can be 
anywhere from 0 to 4 bytes. templates and instructions inherit the properties illustrated in Figure 5.

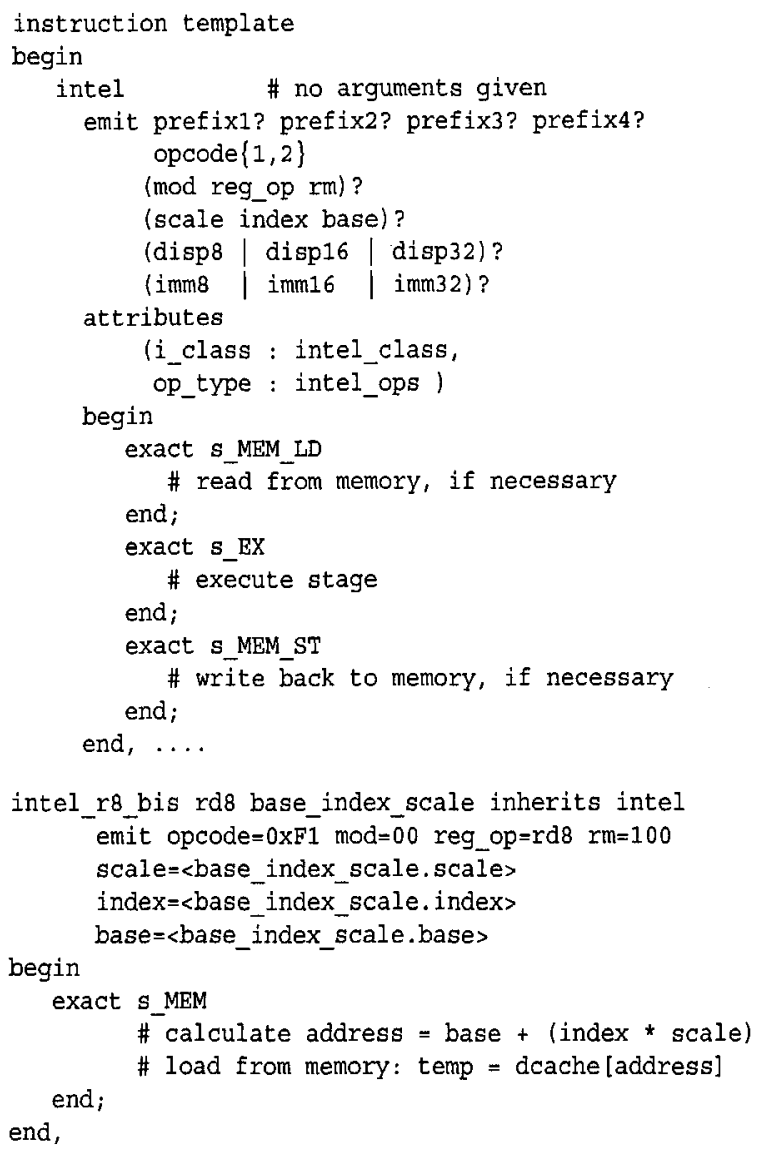

Figure 5. Instruction templates and Using inheritance

The first item to notice is on the $3^{\text {rd }}$ line, no arguments are given to the generic instruction name intel. The non-existent arguments will be overridden by the following templates. The emit line, on the other hand, defines every possible field that might be emitted by a descendant and uses the ? and $\{n, m\}$ modifiers to indicate optional fields. Only two attributes are defined at this time; more will probably be needed when the microarchitecture is implemented. Finally, there are three pipeline stages used to execute the instruction, an execute stage and a memory access before and after the execute stage for those instructions that need it (more on this below). Again, the pipeline stages may change with implementation of the microarchitecture. 
An instruction that inherits from this intel master template is free to override the arguments, any of the emit fields, any of the attributes, or any pipeline stage. (Note that if a pipeline stage is overridden, the entire stage must be overridden, even if only one line is changed.) Inheritance is indicated by the inherits keyword following the instruction's arguments as shown in Figure 5.

The template intel_r8_bis has two arguments, an 8-bit destination register, rd8, and a memory location addressed by base_index_scale mode. It inherits from the intel master template and then defines exactly which fields will be emitted for this type of instruction. The scale, index, and base functions are built-in to the ADL language and, with the help of the regular expressions for the addressing modes, return the respective values for scale, index, and base. Finally, the s_MEM pipeline stage is used to load a byte into a temporary pipeline register which will be used by the S_EX stage in instructions that inherit from this template. (The code to actually load from memory will be specific to how the microarchitecture is implemented, so for now we describe what has to be done in comments.)

Memory Addressing and Conditional Inheritance: Once all the templates are defined as shown above, the final step is to create conditional inheritance. This borrows from the idea of multiple inheritance, except instead of inheriting all of the features from the parents, it only inherits from the one parent with the best fit. The best fit is determined by the arguments to the instruction. (Note that this implies the inherited arguments must be unambiguous.)

This allows us to create one template for each addressing mode. Each template will have the emit fields defined and other common properties. The child that inherits from the template then overrides the emitted opcode field and defines in the pipeline stage what exactly the instruction does (i.e., the semantics that make languages like SLED unfeasible for our work). The common addressing modes are then combined using the conditional inheritance feature into one template which the real instructions will inherit from. To reinforce the idea that this is not traditional multiple inheritance, the $\mid$ operator (logical or) is used to split parents. For example, instructions that have a 32-bit register for a source and a 32-bit word in memory for a destination would inherit from the intel_r32_rm32 template that is shown in Figure 6.

Each of the 18 templates intel_r32.rm32 inherits from define an addressing mode (there are 12 modes plus 6 special modes for using \%esp or \%ebp as a base register). A real instruction then inherits from intel $r 32$ rm32 as shown in Figure 6 . To see this in action, consider the following $x 86$ instructions:

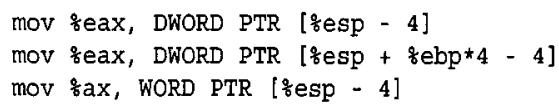

The instruction is the same in both cases, movl, but the arguments differ. However, they differ in a unique and unambiguous way which allow the com- 


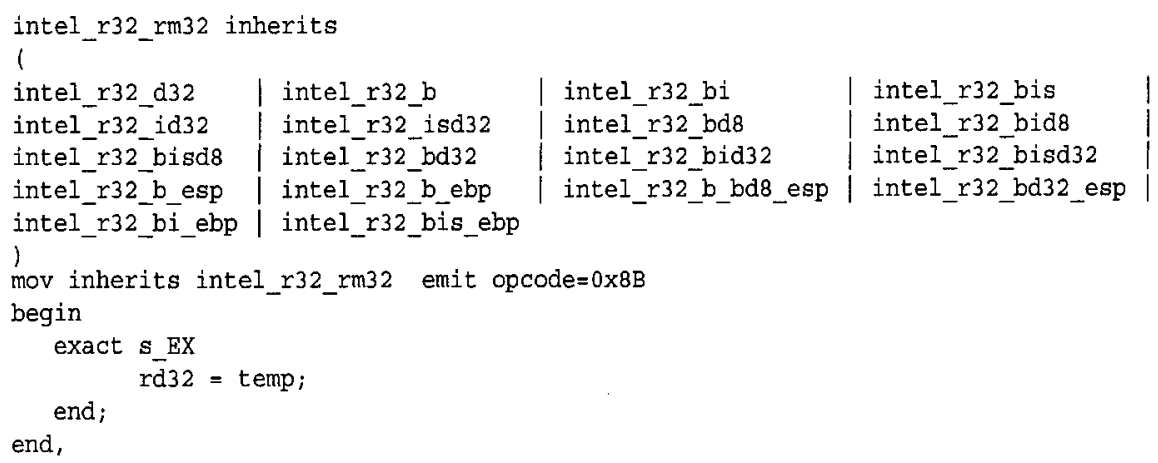

Figure 6. Conditional Inheritance and example instruction using inheritance

piler to match it against only one parent. The first instruction matches intel_r32_rm32 and its parent intel_r32_bd8 (base + 8-bit-displacement) (technically it also matches intel_r32_bd32, but the compiler will be smart enough to choose an 8-bit-displacement if it can via a pragma). Likewise, the second instruction matches intel_r32_rm32 but with a different parent, intel_r32_bisd8. The third instruction matches none of the parents in intel $\mathrm{r} 32 \mathrm{rm} 32$, so the compiler looks for another instruction to match against (which, in this case, will be mov inherits intel_r16_rm16 and its parent intel_r16_bd8).

Overlapping Registers: $\mathrm{C}$ style unions and typesets were introduced to deal with overlapping registers. First, the physical registers are defined as generic 32-bit registers, then a union is used to define the symbolic registers and which parts of the physical register are used in [start bit, length] notation and finally, a typeset is used to group the registers together into logical sets.

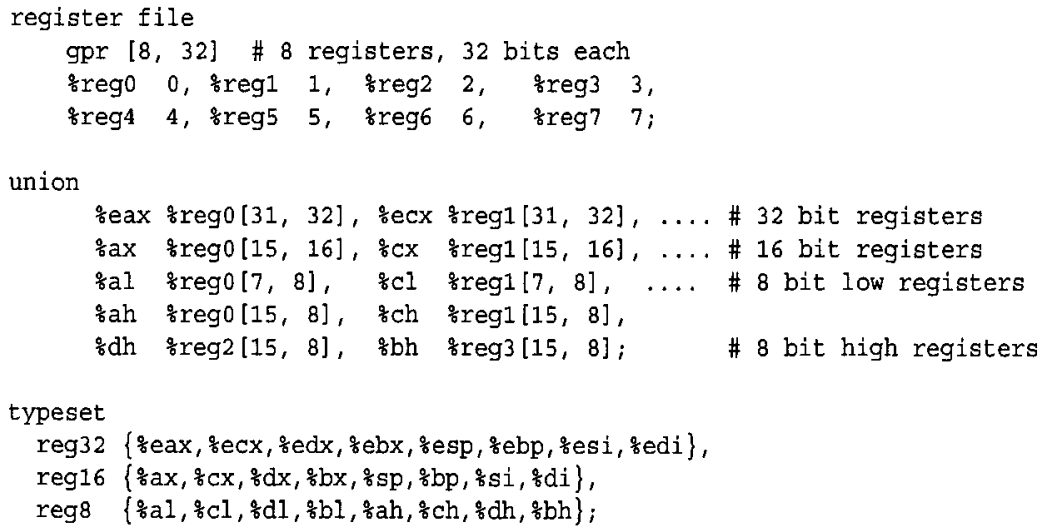

Typesets are also used to place registers into special groups. For example, the \%esp and \%ebp registers cannot be used as a base register in base addressing mode, so a special group is created minus those registers. 


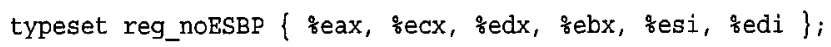

Typesets can be used to group more than just registers. The opcode prefixes are defined as bitconstants and are split into four groups and only one prefix from each group can be used in an instruction. The typeset is used to define the groups.

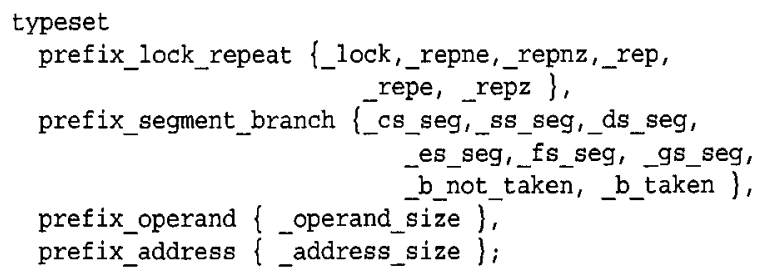

Mixed arguments: The mixed argument problem is also solved through inheritance. The microarchitecture pipeline has not been defined in this paper, but at least three stages will be needed: two memory access stages with an execute stage in the middle. This can be implemented as one major cycle with 3 minor cycles: S_MEM_LD to load from memory, s_EX to execute the instruction, and S_MEM_ST to store the results back to memory.

The instruction templates have code in the load and store minor-cycles that either load from memory into an internal temp register or store the data in temp back to memory. The execute stage uses the temp register instead of the second argument. Thanks to inheritance, this is mostly masked from the final instruction's description. The only concern an instruction has is whether it's working on register-to-register operands or a combination of registers and memory. It can tell which type it is by checking the inherited op_type attribute:

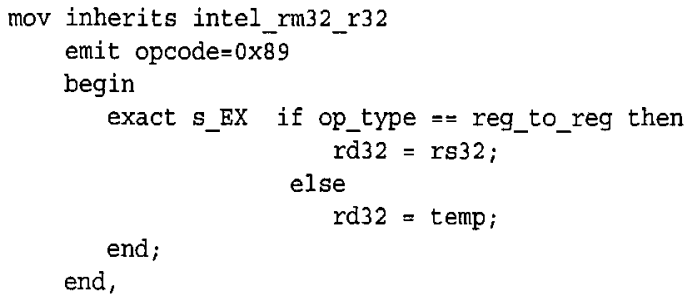

In the above figure, a pipeline with three stages is shown. Real implementations of $x 86$ are typically not pipelined due to the difficulties as seen above (instructions operating directly on memory). In order to pipeline $\mathrm{x} 86$ processors, such as the Pentium, the processor converts the $\mathrm{x} 86$ instruction into a series of micro-operations, and these RISC-like micro-ops are pipelined. Multiple Choice: An interesting feature of the $\mathrm{x} 86$ instruction set is how there is often more than one way to encode an instruction. This is usually due to the 
shortcut opcodes for tasks instructions used often enough to warrant a shortcut. For example, push \%eax, could be encoded two different ways such as [opcode $=0 x F F$ mod=11 regop=110 $\mathrm{rm}=000$ ] as well as [opcode $=0 \times 50$ ].

The push \%eax instruction is used often enough that a special opcode was created just for it. The upside is that it saves a byte of memory. The downside is it presents the assembler with a dilemma on encoding the instruction. Fortunately, the dilemma is resolved easily enough - chose the instruction with the shortest encoding.

\section{Conclusions and Future Work}

The $\mathrm{x} 86$ is a powerful and compact ISA, but it's this same compactness that makes it so difficult to work with (in compilers, in simulators, and more). We have shown that by introducing the notion of conditional multiple inheritance we have tackled the most difficult challenges of $\times 86$ within the realm of an architecture description language.

Our future work on IA-32 on FAST can be broken into two broad areas, namely the implementation of the language constructs in the ADL compiler and the completion of the micro-architecture specification. Once running simulators are successfully generated from the ADL specification, we are planning to remove the restrictions and add the MMX and SSE/SSE2 instructions to the ISA. 


\section{References}

[1] J.R. Armstrong and F.G. Gray. Structured Logic Design with VHDL. New Jersey: Prentice Hall, 1993.

[2] D.C. Burger and T.M. Austin. The SimpleScalar Tool Set, V. 2.0. Technical Report 97-1342, Computer Sci. Dept., Univ. of Wisconsin Madison, 1997.

[3] J.R. Larus. SPIM S20: A MIPS R2000 Simulator. Technical Report 90-966, Computer Sci. Dept., Univ. of Wisconsin Madison, 1990.

[4] J.D. Morison and A.S. Clarke. ELLA2000 A language for Electronic System Design. McGraw-Hill, 1993.

[5] C. Moura. SuperDLX a generic superscalar simulator. Technical Report 64, School of Computer Science, McGill University, 1993.

[6] Soner Önder and Rajiv Gupta. Automatic generation of microarchitecture simulators. In IEEE International Conference on Computer Languages, pages 80-89, Chicago, May 1998.

[7] Robert Pastel. Describing vliw architectures using a domain specific language. Master's thesis, Michigan Technological University, 2001.

[8] D.L. Perry. VHDL. McGraw-Hill, 1991.

[9] David Poplawski. The unlimited resource machine (urm). Technical report, Michigan Technological University, 1995.

[10] Norman Ramsey and Mary F. Fernandez. The new jersey machine-code toolkit. In Proceedings of the 1995 USENLX Technical Conference, New Orleans, LA, pages 289302, January 1995.

[11] Norman Ramsey and Mary F. Fernandez. Specifying representations of machine instructions. ACM Transactions on Programming Languages and Systems, 19(3):492-524, May 1997.

[12] D.E. Thomas and P.R. Moorby. The Verilog Hardware Description Language. Kluwer Academic Publishers, 1991. 